\title{
Disseminated Cryptococcosis in an HIV-negative patient in South Africa: the elusive differential diagnosis
}

\author{
Mohammed Mitha ${ }^{1}$, Preneshni Naicker ${ }^{2}$, Prakash Mahida ${ }^{1}$ \\ ${ }^{1}$ Department of Internal Medicine and ${ }^{2}$ Department of Medical Microbiology, Tygerberg Hospital, University of \\ Stellenbosch, Cape Town, South Africa
}

\begin{abstract}
The presence of an opportunistic infection in a patient in sub-Saharan Africa is assumed to be due to underlying immunosuppression from human immunodeficiency virus (HIV) infection. The presence of disseminated cryptococcosis in a non-HIV-infected patient is interesting as it is unique in our setting because the majority of infections are found in HIV-infected individuals. The protean manifestations of the disease and its predilection for immunosuppressed patients make cryptococcosis a challenging and elusive disease to diagnose in HIV-negative patients in our setting, especially due to limited resources. We present a case of disseminated cryptococcosis in an immunocompetent patient and discuss diagnostic and therapeutic features in this subset of patients.
\end{abstract}

Key words: cryptococcosis; immunocompetent; South Africa

J Infect Dev Ctries 2010; 4(8):526-529.

(Received 05 January 2010 - Accepted 08 March 2010)

Copyright (c) 2010 Mitha et al. This is an open-access article distributed under the Creative Commons Attribution License, which permits unrestricted use, distribution, and reproduction in any medium, provided the original work is properly cited.

\section{Introduction}

The number of people estimated to be infected with HIV in sub-Saharan Africa is 22.5 million, which is approximately two thirds (67\%) of the global population infected with HIV. The HIV epidemic in South Africa has been described as a hyperendemic epidemic according to the Joint United Nations Programme on HIV/AIDS [1,2].

Cryptococcal disease is frequently seen in South Africa in HIV-infected patients with meningitis as the commonest presentation. The incidence is estimated at 114/100.000 cases of HIV-infected individuals and 10/1.000 in those with AIDS [1].

The clinical features of cryptococcosis differ between HIV-infected and non-infected persons. Meningoencephalitis is predominant in HIV-positive patients, whereas HIV-negative patients generally present with pulmonary or central nervous system (CNS) mass lesions [3].

Cryptococcosis in immunocompetent persons is rarely diagnosed, and the incidence and prevalence of cryptococcal disease in non HIV-infected persons is unknown in South Africa. Reasons for infrequent diagnosis in uninfected individuals may include the fact that the disease actually is rare and therefore not considered by health professionals, and the lack of resources to ascertain tissue specimens when the diagnosis is considered. As a consequence, diagnosis is also frequently delayed, resulting in complications. The treatment protocols differ between Cryptococcus species, sites of infection, and immune status.

\section{Case report}

In July 2008, a 40-year-old HIV-negative male patient was referred to Tygerberg Hospital, a tertiary level hospital in Cape Town, South Africa, after presenting with a right-sided hemiparesis and background history of two generalised tonic-clonic seizures which started in May 2008. The patient was previously healthy with no background medical history, no previous history of tuberculosis (TB), no history of head trauma, and he was of sober habits. $\mathrm{He}$ did not have any constitutional symptoms and there was no significant exposure to pigeons. His respiratory, cardiovascular, and abdominal examinations were normal.

A computed tomography (CT) scan demonstrated a large multiloculated ring enhancing lesion in the left frontal lobe with associated vasogenic oedema with a smaller lesion noted in the right frontal region. The lesion was suspected of being a gummatous 
tuberculoma and the patient was commenced on antiTB therapy and steroids.

A CT brain scan was repeated after the patient had been on anti-TB treatment three months, which demonstrated marked progression of the previously ring enhancing lesions as well as development of at least two new lesions (Figure 1). There was a midline shift to the right. The patient tested negative for HIV, syphilis, and toxoplasmosis at this stage. There were no immunoglobulin deficiencies, and the autoimmune screen was negative. His CD4 count was 420 cells $/ \mu 1$.

Figure 1. Contrast enhanced CT scan of the brain demonstrating cryptococcomas as well as midline shift.

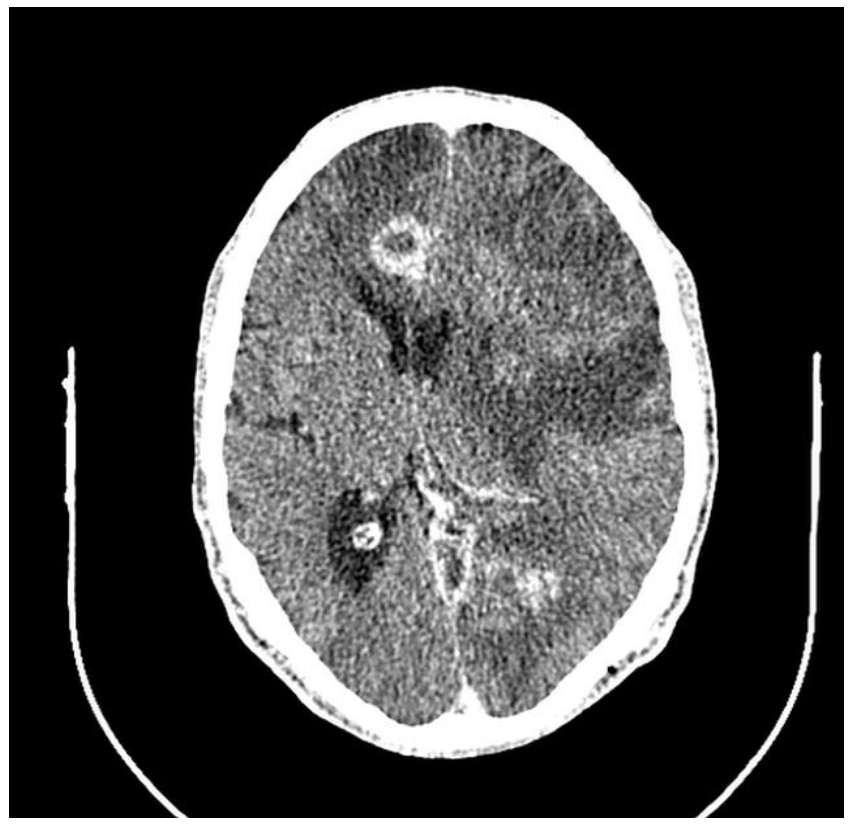

A six-month follow-up revealed a worsening right hemiparesis. An MRI of the brain showed lesions suggestive of metastases. A chest radiograph showed a retro-cardiac mass suggestive of bronchial carcinoma. A CT chest demonstrated a cystic mass in the left lower lung zone.

A trans-bronchial needle aspirate confirmed the diagnosis of the yeast fungus Cryptococcus neoformans var. gattii on culture and histology. A brain biopsy also confirmed cryptococcomas. A serum cryptococcal latex agglutination test was negative. The disease was definitively diagnosed in February 2009, seven months after initial presentation.
The patient was commenced on Amphotericin B $1 \mathrm{mg} / \mathrm{kg}$ daily but developed renal impairment. Therapy was changed to Fluconazole (400 mg every 12 hours) after receiving Amphotericin B for fourteen days. The organism was susceptible to both these antifungal agents.

A CT brain scan was repeated in April 2009 which demonstrated no regression of the lesions. The patient was then lost to follow-up and could not be further traced.

\section{Discussion}

Cryptococcal disease in humans is caused principally by two species in the Cryptococcus genus, namely Cryptococcus neoformans and Cryptococcus gattii (Cryptococcus neoformans var. gattii.) [3]. Cryptococcus neoformans is found worldwide, commonly in pigeon excrement in soil, and it causes disease in immunompromised hosts. Cryptococcus gattii has recently been recognised as a distinct emerging pathogen causing disease in humans and animals. It is found predominantly on eucalyptus trees in tropical and subtropical regions and causes disease in immunocompetent individuals [1,3,4].

The organism is inhaled into the respiratory tract and, depending on the immune response, the host may be asymptomatic or disease may occur presenting either as pneumonitis, pulmonary nodules, or less commonly as pleural effusions [3]. The most common radiographic feature is pulmonary nodules and mass lesions both on chest radiograph and CT scan $[5,6]$. After primary infection in the lung, the organism can spread to distant sites causing disease; however, this is more common in immuncompromised individuals [3].

The organism most frequently affects the central nervous system (CNS), causing meningitis. However, it rarely affects the parenchyma causing cryptococcomas which tend to present with focal signs [3,7]. The presentation of CNS disease is generally subacute and the diagnosis is made late, as was evident in the case, approximately seven months from the onset of symptoms $[7,8,9]$.

Other immunosuppressive conditions, such as organ transplantation, malignancy, chronic renal failure and diabetes mellitus, may predispose an individual to cryptococcosis [5]. In approximately $10-40 \%$ of patients, no underlying immunosuppressive state can be noted [5,8].

The diagnosis was delayed in this case because cryptococcosis was not considered as a differential diagnosis due to the patient's negative HIV status. A 
malignancy was suspected in view of the chest radiograph and MRI findings. This case demonstrates that cryptococcosis should be included as part of the differential diagnosis for isolated pulmonary lesions seen on chest radiographs. Serum cryptococcal antigen assay may also aid in the diagnosis, especially when a lumbar puncture is contraindicated. In this case, a lumbar puncture could not be performed due to raised intra-cranial pressure. The cryptococcal antigen assay was negative in this patient; however, a negative serum assay is rare in cryptococcosis but it may occur in immunocompetent patients and those with cryptococcomas [10]. A fungal blood culture can also be utilized, but the yield is reduced in immunocompetent patients [5].

The treatment of cryptococcosis is based on the Infectious Diseases Society of America guidelines which depend on the patient's immune profile, the infected organ systems, and the species type. The treatment is the same for both $C$. neoformans and $C$. gattii with regard to CNS and disseminated disease. The aim of treatment in CNS lesions is sterilization and to prevent neurological sequelae. The treatment is generally prolonged, requiring up to six weeks of intravenous therapy depending on the availability of medications, followed by a further six to 18 months of oral treatment. In certain cases, surgery may be required [11]. These guidelines have been based on retrospective data from $\mathrm{HIV}$-infected patients and there have been few randomized controlled trials addressing the management of cryptococcosis in immunocompetent patients [5,11]. Further studies are required in this field, as certain medications are not available and 6 weeks of intravenous medication is extremely difficult in our setting due to frequent bed shortages and limited resources. The need for longterm therapy also poses a major challenge on limited government resources.

Patients must be followed up closely and reviewed frequently to monitor treatment progress. There may be a compliance issue with regard to the long treatment duration and the patients must be adequately counselled. Diagnostic radiology is a useful tool to assist in the management of cerebral cryptococcomas, especially those caused by $C$. gattii. Repeat scans are needed to objectively determine the response to treatment by demonstrating regression of the lesions. Patients much have repeat CT scans to determine response to treatment. It may be advisable to repeat the scan after three months after initiation of treatment, and subsequently every three months thereafter. However, due to the cost of CT scans, this strategy must be determined by further research for cost effectiveness.

The reason for infection in this case was undetermined, as it is in one third of cases [5]. However, large inoculum size is a possibility, as has been postulated in cases of a similar nature [12]. The patient may have been exposed to $C$. gattii from eucalyptus trees in his district and it may be of interest to determine the prevalence of cryptococcus on eucalyptus trees in the Western Cape province of South Africa.

Cryptococcosis in immunocompetent patients in South Africa is either rare or is being underdiagnosed due the low index of suspicion in HIV-negative individuals. The diagnosis may also be difficult to determine due to limited resources. A differential diagnosis of cryptococcal disease should be considered in all patients presenting with either pulmonary or CNS lesions or both, irrespective of their immune profile. A serum cryptococcal antigen test should be done on all patients with pulmonary lesions as well as mass lesions in the CNS. If this approach is employed, diagnoses will be made sooner and treatment commenced earlier, which may possibly prevent severe morbidity and mortality.

Infection due to $C$. gattii can pose a diagnostic challenge to clinicians. This case vividly illustrates that pulmonary cryptococcosis with a mass-like lesion with associated cerebral infection exhibits radiological features that mimic those of lung cancer with metastatic disease to the CNS. The physician must be cognisant at all times of other causes and must try to ascertain the diagnosis. Although TB is common in South Africa, this rare entity of CNS cryptococcoma in an HIV-negative patient has clearly demonstrated that this differential diagnosis should not be disregarded because of the patient's immune status.

\section{References}

1. Department of Health, Republic of South Africa: The burden of cryptococcosis in South Africa. Statistical Notes February 2008. Available: http://www.doh.gov.za/docs/stats/2008/cryptococcosis.Acce sed 30 June 2009

2. South African National HIV Prevalence, Incidence and Behaviour and Communication Survey 2008. Human Sciences Research Council. Available: http://www.hsrcpress.ac.za Accessed 30 March 2010

3. King JW Cryptococcosis (2009) eMedicine. Available: http://www.emedicine.com/med/topic 482. Accessed 30 October 2009.

4. Lin X (2009) Cryptococcus neoformans: Morphogenesis, infection, and evolution. Infection, Genetics and Evolution. 9: 401-416. 
5. Kiertiburanakul S, Wirojtananugoon S, Pracharktam R,Sangkanuparph S $l$ (2006) Cryptococcosis in human immunodeficiency virus negative patients. Int $\mathbf{J}$ of Infects Dis 10: 72-78.

6. Yang CJ, Hwang JJ, Wang TH, Cheng MS, Kang WY, Chen TC, Huang MS (2006)Clinical and radiographic presentations of pulmonary cryptococcosis in immunocompetent patients. Scand J Infect Dis 38: 788.

7. Cox GM and Perfect JR (1997) Cryptococcus neoformans var neoformans and gattii and Trichosporon species. In Ajello, E editor. Topley and Wilson's Microbiology and Microbial Infections, 9th ed. London: Arnold Press.p 461-484

8. Lui G, Lee N, Ip M, Choi KW, Tso YK, Lam E, Chan S, Lai R, Cockram CS (2006) Cryptococcosis in apparently immunocompetent patients. Q J Med 99: 143-151.

9. Ecevit IZ, Clancy CJ, Schmalfuss IM, Hong Nguyen M $l$ (2006) The poor prognosis of central nervous system cryptococcosis among nonimmunosuppressed patients: a call for better disease recognition and evaluation of adjuncts to antifungal therapy. Clin Infect Dis 42: 1443-1447.

10. Eric Searls D, Sico JJ, Bulent Omay S, Bannykh S, Kuohung V,Baehring J (2009) Unusual presentations of nervous system infection by Cryptococcus neoformans. Clin Neurol Neurosurg 111: 638-642.

11. Perfect JR, Dismukes WE, Dromer F, Goldman DL, Graybill JR, Hamill RJ, Harrison TS, Larsen RA, Lortholary O, Nguyen MH, Pappas PG, Powderly WG, Singh N, Sobel
JD, Sorrell TC (2010) Clinical Practice Guidelines for the Management of Cryptococcal Disease: 2010 Update by the Infectious Diseases Society of America. Clin Infect Dis 50: 291-322.

12. Saigal G, Donovan Post MJ, Lolayekar S, Murtaza A Unusual presentation of central nervous system cryptococcal infection in an immunocompetent patient. Am J Neuroradiol 26: $2522-2526$.

\section{Corresponding author}

Mohammed Mitha

Registrar in Internal Medicine

Tygerberg Hospital

University of Stellenbosch

Cape Town, South Africa

75 Devon Terrace

Westville

3630

South Africa

Tel (mobile): +27 823436821

Fax: +27 312669089

Email: momitha@telkomsa.net

Conflict of interests: No conflict of interests is declared. 